\title{
SIRT1 exerts neuroprotective effects by attenuating cerebral ischemia/reperfusion-induced injury via targeting p53/microRNA-22
}

\author{
HUI LU ${ }^{1}$ and BINCHENG WANG ${ }^{2}$ \\ ${ }^{1}$ Department of Neurology, Cangzhou Central Hospital, Hebei 060000; \\ ${ }^{2}$ Department of Neurology, Xuan Wu Hospital, Beijing 100010, P.R. China
}

Received December 28, 2015; Accepted October 4, 2016

DOI: $10.3892 / \mathrm{ijmm} .2016 .2806$

\begin{abstract}
The aim of this study was to investigate whether the SIRT1 exerts neuroprotective effects by attenuating cerebral ischemia/reperfusion-induced injury (CIRI) via targeting p53/microRNA-22. We found that the overexpression of sirtuin 1 (SIRT1) decreased the infarct volume, suppressed p53 protein expression and activated microRNA-22 expression following CIRI. An injection of lipopolysaccharide (LPS, $1 \mathrm{mg} / \mathrm{ml}$; Sigma, St. Louis, MO USA) into the corpus callosum was used to induce CIRI in rats. The infarct volume and neurological deficit score were used to examine the effects of SIRT1 on CIRI. Furthermore, the overexpression of SIRT1 was found to suppress caspase-3 activity, inhibit the activation of the Bax signaling pathway, reduce tumor necrosis factor- $\alpha(\mathrm{TNF}-\alpha)$ and interleukin (IL)-6) activity, decrease cyclooxygenase (COX)-2 and inducible nitric oxide synthase (iNOS) protein expression, and increase IL-10 activity following CIRI. Following the downregulation of SIRT1, p53 protein expression was significantly increased, microRNA-22 expression was inhibited, caspase-3 activity was increased and the Bax signaling pathway was activated. In addition, the activity of TNF- $\alpha$ and IL- 6 was was enhanced, COX-2 and iNOS protein expression was increased, and IL-10 activity was reduced following CIRI. Thus, the data from our study suggest that SIRT1 attenuates CIRI by targeting the p53/microRNA-22 axix, while suppressing apoptosis, inflammation, COX-2 and iNOS expression.
\end{abstract}

\section{Introduction}

Clinically, acute ischemic cerebrovascular disease is very common with high morbidity, mortality and disability (1). Cerebral ischemia can trigger nerve cell damage (1). The funda-

Correspondence to: Mr. Hui Lu, Department of Neurology, Cangzhou Central Hospital, Wantai Landscape Area 3-1-2403, Canal Zone Cangzhou, Hebei 060000, P.R. China

E-mail:du63496@126.com

Key words: sirtuin 1, cerebral ischemia/reperfusion-induced injury, p53, microRNA-22 mental measures for preventing ischemic injury are to recover the blood supply as soon as possible (2). With the recovery of blood perfusion to the ischemic area after continuing cerebral ischemia for a certain period of time, the original cells at the ischemic area cannot recover (2). Thus, this aggravates ischemic injury. This phenomenon is known as cerebral ischemia/ reperfusion injury (CIRI) (3). The pathogenesis of CIRI is more complex than that of pure hypoxic-ischemic brain damage and is associated with nerve cell apoptosis, the breakdown of high energy phosphate compounds, lipid peroxidation, inflammatory reactions, the toxic effects of excitatory amino acids, calcium overload, the functions of nitric oxide (NO) and endothelial cell dysfunction (4-6). However, the specific mechanisms responsible for the pathogenesis of CIRI have not yet been fully elucidated.

Sirtuin 1 (SIRT1) is a type of histone deacetylase dependent on $\mathrm{NAD}^{+}$, which is the highest factor in homologous genes of mammals in silent information regulator SIR2 (7). Studies have confirmed that SIRT1 is extensively expressed in tissues and organs and is associated with metabolic syndrome, tumors and neurodegenerative diseases (8-10). A previous study demonstrated that icariin exerted neuroprotective effects through the induction of SIRT1, and that these effects were reversed by the use of a SIRT1 inhibitor (11). Moreover, SIRT1 can regulate many transcription factors and receptors, such as hypermethylated in cancer 1 (HIC1), C-terminal-binding protein (CtBP), p53, forkhead box O3 (FOXO3A) and E2F transcription factor 1 (E2F1) $(9,11,12)$. The hippocampus is a key structure of learning and memory in the brain. The expression of SIRT1 in the brain is the material basis for its participation in regulating cognitive functions (13). SIRT1 plays a role in neuronal differentiation (13). It has also been shown that SIRT1 participates in the dendronization of neuronal dentritic cells (11). The overexpression of SIRT1 in nurtured hippocampal neurons enhances the dendronization of dendrites (14).

During the later decades of an individual's life, the morbidity and death rates triggered by cerebral ischemia are relatively high. Owing to delayed neuronal death (DND), despite survival after an attack, nervous system sequelae, such as motor dysfunction, and language and cognitive deficits may occur (15). It has been proven that apoptosis is closely associated with DND. Some apoptosis-related genes have also been found. The tumor suppressor gene, p53, has been found to participate in the DND 
of brain neurons following ischemia. It has dual functions in cerebral ischemia (16). When DNA is damaged, p53 causes cell cycle arrest, which helps the repair of DNA. When DNA cannot be restored, it can induce cell apoptosis (17).

During the process of ischemia/reperfusion, many genes undergo alterations, resulting in the inhibition of some proteins, while the expression of other harmful proteins is significantly upregulated (18). As important components in genetic expression, the levels and functions of mRNAs are regulated by several factors. Studies have found that microRNAs participate in various biological process, such as embryogenesis, proliferation, differentiation and cell death $(19,20)$. Thus, it has been predicted that microRNAs regulate genec expression in over a third of animals. In diseases of the nervous system, the abnormal expression of microRNAs is associated with the dysfunction of synaptogenesis and synaptic reorganization, degenerative diseases, tumors and epilepsy $(18,20)$. A recent study indicated that microRNAs (microRNA-107) participate in the development of CIRI (21). In the present study, therefore, we wished to determine whether SIRT1 exerts neuroprotective effects by attenuating CIRI and explored the mechanisms involved.

\section{Materials and methods}

Animals, model of CIRI and drug treatment. Sprague-Dawley male rats $(\mathrm{n}=56$, weighing, 230-260 g) were purchased from Founder Animal's Pharmaceutcal Co., Ltd. (Cangzhou, China) and kept $\left(22 \pm 1^{\circ} \mathrm{C}, 55 \pm 5 \%\right.$ humidity) on a $12 \mathrm{~h}$ light/dark cycle with ad libitum access to food and water. All experimental procedures were approved by the Committee of Cangzhou Central Hospital in accordance with the NIH Guide for the Care and Use of Laboratory Animals. The rats were anesthetized with chloral hydrate [300 mg/kg, intraperitoneal (i.p.) injection]. LPS ( $1 \mathrm{mg} / \mathrm{ml}$; Sigma, St. Louis, MO USA) was injected into the corpus callosum in order to induce CIRI. After 1 day, focal cerebral ischemia was induced by right-sided endovascular middle cerebral artery occlusion. The suture was pulled back and the rats were allowed to recover after $2 \mathrm{~h}$ of ischemia. Firstly, 32 rats were randomly assigned into 4 groups as follows: i) the shamoperated (control, $\mathrm{n}=8$ ) group; ii) the vehicle group (CIRI model, $\mathrm{n}=8$ ); iii) the group treated with $5 \mathrm{mg} / \mathrm{kg}$ of sc-222315 (SIRT1 activator 3, $\mathrm{n}=8$; chemical structure shown in Fig. 1A); and iv) the group treated with $10 \mathrm{mg} / \mathrm{kg}$ group of sc-222315 $(\mathrm{n}=8)$. A total of 16 rats were treated with SIRT1 activator 3 for 48 h by intraperitoneal injection. Subsequently, 24 rats were randomly assigned into 3 groups as follows: i) the sham-operated (control, $\mathrm{n}=8$ ) group; ii) the vehicle group (CIRI model, $\mathrm{n}=8$ ); and the group treated with $10 \mathrm{mg} / \mathrm{kg}$ of sirtinol (S7942; SIRT1 inhibitor, $\mathrm{n}=8$; chemical structure shown in Fig. 1B). A total of 8 rats were treated with sirtinol for $48 \mathrm{~h}$ by intraperitoneal injection. The rats were then anesthetized with chloral hydrate $(300 \mathrm{mg} / \mathrm{kg}$, by intraperitoneal injection) and sacrificed by decollation. The brains were immediately removed for determining the infarct volume, and the brain tissue samples were washed with PBS and stored at $-80{ }^{\circ} \mathrm{C}$ until use.

Measurement of infarct volume and neurological deficit score. The brain tissue samples were cut into coronal slices of $2 \mathrm{~mm}$ for determining the infarct volume using $2 \%$ triphenyltetrazolium chloride (TTC; Sigma). The integrating 6 selected sections were

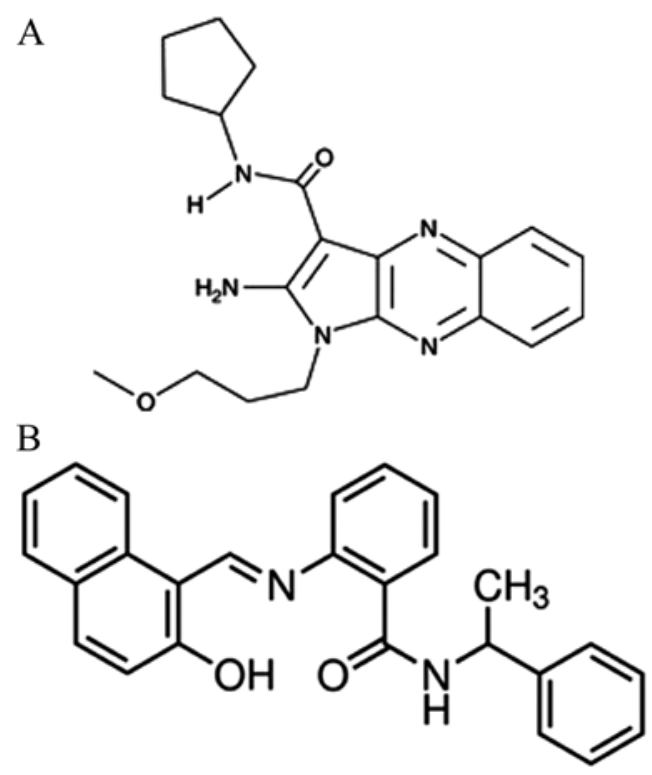

Figure 1. Chemical structure of SIRT1 activator 3 and sirtinol. The chemical structure of (A) SIRT1 activator 3 and (B) sirtinol.

used to calculate the infarct volume, and the corrected infarct volumes were calculated to compensate for brain edema. The neurological deficit score was analyzed based on the following criteria and five-point scoring system: no neurological injury symptoms was given a score of 0 ; inability to entirely extend the front jaw on the heterolateral side was given a score of 1 ; rotation while crawling and falling to the heterolateral side was given a score of 2; inability to walk without assistance was given a score of 3; unconsciousness was given a score of 4 .

Measurement of inflammation and caspase -3 activity. Brain samples were homogenized in $50 \mathrm{mM}$ Tris buffer and were then subjected to centrifugation at $12,000 \mathrm{x}$ g for $10 \mathrm{~min}$ at $4^{\circ} \mathrm{C}$ for determining the activity of tumor necrosis factor- $\alpha$ (TNF- $\alpha$ ), interleukin (IL)-6, IL-10, and caspase-3 in the rat brains using ELISA kits in accordance with the manufacturer's instructions (Elabscience Biology Co., Ltd., Wuhan, China).

Western blot analysis. The brain samples were homogenized in $50 \mathrm{mM}$ Tris buffer, and the protein concentration in the supernatant was determined using the Micro BCA protein assay kit with bovine serum albumin as the standard (Pierce, Rockford, IL, USA) following centrifugation at $12,000 \mathrm{x}$ g at $4^{\circ} \mathrm{C}$ for $10 \mathrm{~min}$. Total protein $(50 \mu \mathrm{g})$ was subjected to $10-12 \%$ sodium dodecyl sulfate (SDS)-polyacrylamide gel electrophoresis and transferred onto polyvinylidene difluoride membranes (Millipore, Bedford, MA, USA). The membranes were incubated with $5 \%$ non-fat dry milk in Tween-20 (0.1\%)-containing TBS for $1 \mathrm{~h}$, followed by incubation with primary antibodies against cyclooxygenase (COX)-2 (sc-7951), inducible NO synthase (iNOS; sc-650), p53 (sc-47698) and $\beta$-actin (sc-47778) (all from Santa Cruz Biotechnology, Inc., Santa Cruz, CA, USA) at $4^{\circ} \mathrm{C}$ overnight. The membranes were washed with TBST and then incubated with anti-mouse or anti-rabbit secondary antibody (sc-2354 or sc-2768; Santa Cruz Biotechnology, Inc.) and examined using the ECL kit (Amersham, Piscataway, NJ, USA). 

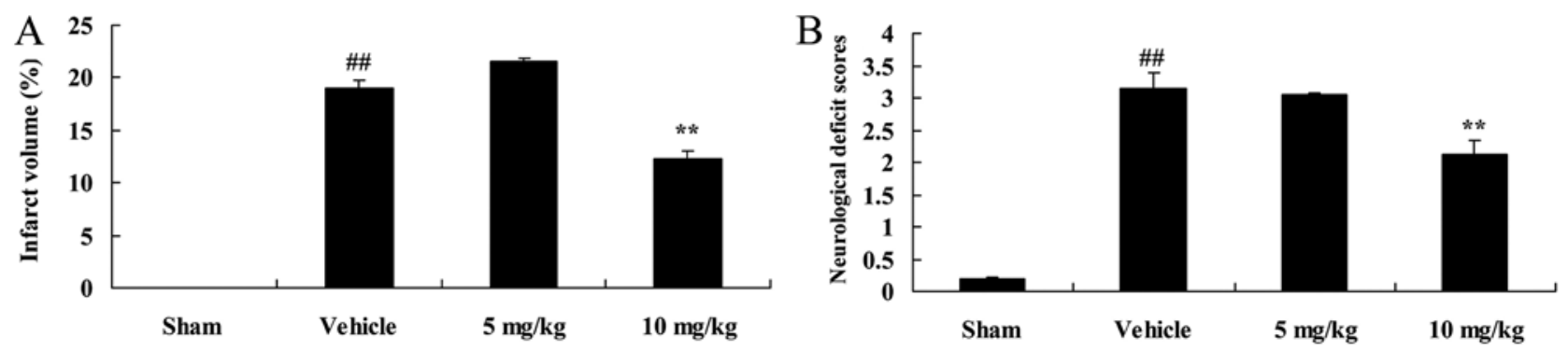

Figure 2. SIRT1 decreases the infarct volume and neurological deficit score in rats with cerebral ischemia reperfusion-induced injury (CIRI). SIRT1 decreases the (A) infarct volume and (B) neurological deficit score in rats with CIRI. Sham, sham-operated group; vehicle, CIRI model rat group; $5 \mathrm{mg} / \mathrm{kg}$, group treated with $5 \mathrm{mg} / \mathrm{kg}$ of SIRT1 activator 3; $10 \mathrm{mg} / \mathrm{kg}$, group treated with $10 \mathrm{mg} / \mathrm{kg}$ of SIRT1 activator 3. ${ }^{\# \#} \mathrm{p}<0.01$ compared with sham group; ${ }^{* *} \mathrm{p}<0.01 \mathrm{compared}$ with vehicle group.

Real-time PCR. Total RNA was extracted from the brain samples using TRIzol reagent according to the manufacturer's instructions (Sigma). The gene expression of microRNA-22 was detected by TaqMan MicroRNA assays using loopedprimer reverse transcription (RT)-PCR. The conditions for PCR were as follows: at $95^{\circ} \mathrm{C}$ for $10 \mathrm{~min}$; at $95^{\circ} \mathrm{C}$ for $30 \mathrm{sec}$ and $60^{\circ} \mathrm{C}$ for $30 \mathrm{sec}$ followed by 40 cycles. Glyceraldehyde 3-phosphate dehydrogenase (GAPDH) was used as an internal control. The sequences of the primers used were as follows: GAPDH sense, 5'-GAAGGTGAAGGTCGGAGTC-3' and antisense, 5'-GAAGATGGTGATGGGATTTC-3'; and microRNA-22 sense, 5'-AACTCGAGCGAGTCCTCTCC TAGGACTA-3' and antisense, 5'-TTGCGGCCGCACAC AAAGCTTAAATATG-3'.

Statistical analysis. Data are expressed as the means \pm SD and analyzed for statistical significance using one-way ANOVA, followed by Scheffe's test for multiple comparisons. A value of $\mathrm{P}<0.05$ was considered to indicate a statistically significant difference.

\section{Results}

SIRT1 decreases the infarct volume and improves the neurological deficit score in a rat model of CIRI. Initially, we examined the effects of SIRT1 on the infarct volume and neurological deficit score in rats with CIRI. As shown in Fig. 2, the rats with CIRI exhibited a significant increase in infarct volume and the neurological deficit score, compared with the rats in the sham-operated group. However, treatment with $10 \mathrm{mg} / \mathrm{kg}$ of SIRT1 activator 3 significantly attenuated the CIRI-induced increase int he infarct volume and neurological deficit score in the rats (Fig. 2).

SIRT1 attenuates inflammation in a rat model of CIRI. We then examined the neuroprotective effects of SIRT1 on inflammatory reactions in our rat model of CIRI. The activity of TNF- $\alpha$ and II- 6 was significantly enhanced, and that of IL-10 was significantly inhibited following CIRI, compared with the sham-operated group (Fig. 3). However, treatment with $10 \mathrm{mg} / \mathrm{kg}$ of SIRT1 activator 3 significantly suppressed the activity of TNF- $\alpha$ and II-6, and increased that of IL-10 in the rats with CIRI (Fig. 3).

SIRT1 decreases COX-2 protein expression in a rat model of CIRI. Furthermore, to clarify the regulatory mechanisms of
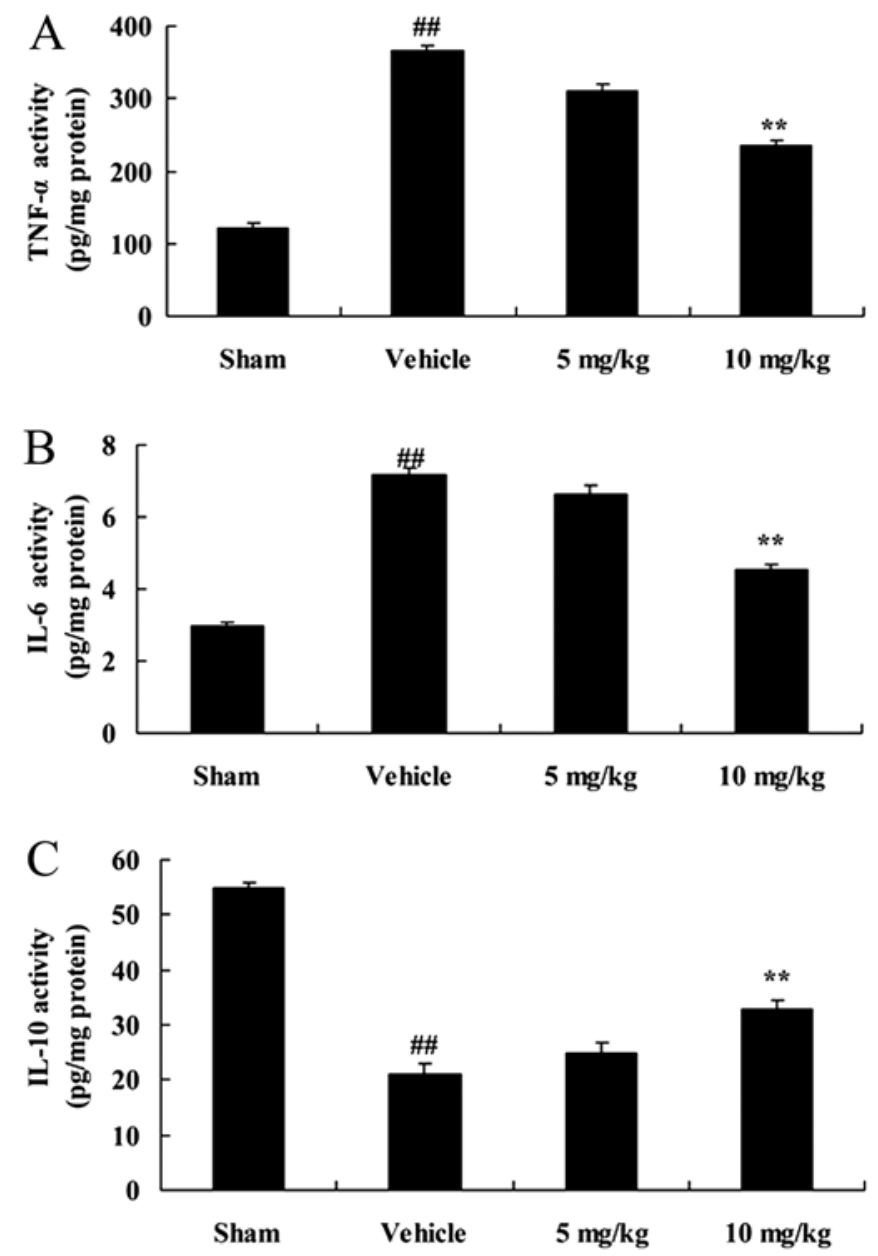

Figure 3. SIRT1 attenuates inflammation in rats with cerebral ischemia reperfusion-induced injury (CIRI). SIRT1 decreased the activity of (A) tumor necrosis factor- $\alpha$ (TNF- $\alpha$ ), (B) interleukin (II)-6 and (C) IL-10 in rats with CIRI. Sham, sham-operated group; vehicle, CIRI model rat group; $5 \mathrm{mg} / \mathrm{kg}$, group treated with $5 \mathrm{mg} / \mathrm{kg}$ of SIRT1 activator $3 ; 10 \mathrm{mg} / \mathrm{kg}$, group treated with $10 \mathrm{mg} / \mathrm{kg}$ of SIRT1 activator $3 .{ }^{\# \#} \mathrm{p}<0.01$ compared with sham group; ${ }^{* *} \mathrm{p}<0.01$ compared with vehicle group.

SIRT1 in rats with CIRI, we first examined COX-2 protein expression in our rat model of CIRI. As shown in Fig. 4, CIRI significantly increased the protein expression of COX-2 in the rats with CIRI, compared with those in the sham-operated group. The induction of COX-2 protein expression following 
A

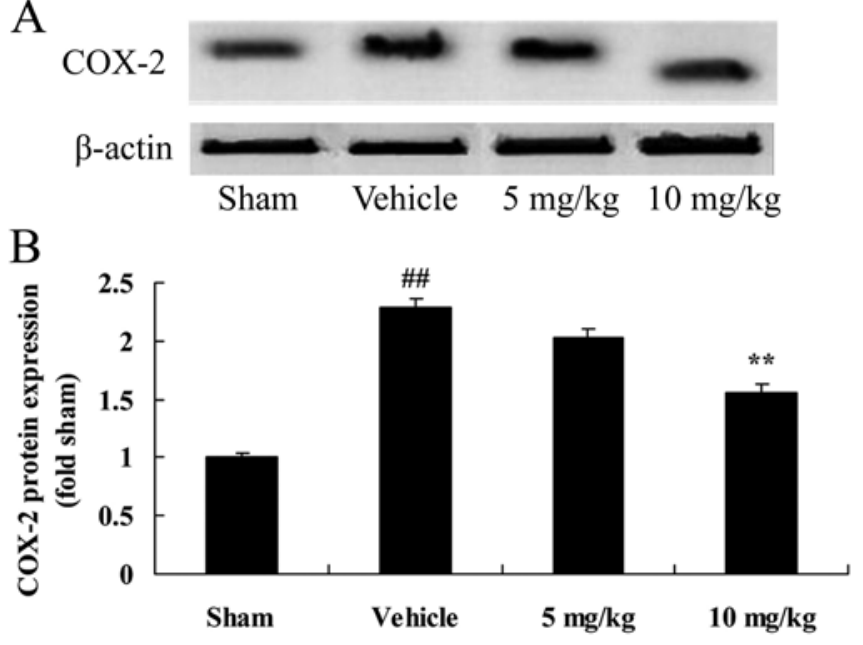

Figure 4. SIRT1 decreases COX-2 protein expression in rats with cerebral ischemia reperfusion-induced injury (CIRI). SIRT1 decreased COX-2 protein expression, as shown by (A) western blot analysis and (B) statistical analysis of COX-2 protein expression in rats with CIRI. Sham, sham-operated group vehicle, CIRI model rat group; $5 \mathrm{mg} / \mathrm{kg}$, group treated with $5 \mathrm{mg} / \mathrm{kg}$ of SIRT1 activator $3 ; 10 \mathrm{mg} / \mathrm{kg}$, group treated with $10 \mathrm{mg} / \mathrm{kg}$ of SIRT1 activator 3 . ${ }_{\# \#} \mathrm{p}<0.01$ compared with sham group; ${ }^{* *} \mathrm{p}<0.01$ compared with vehicle group.

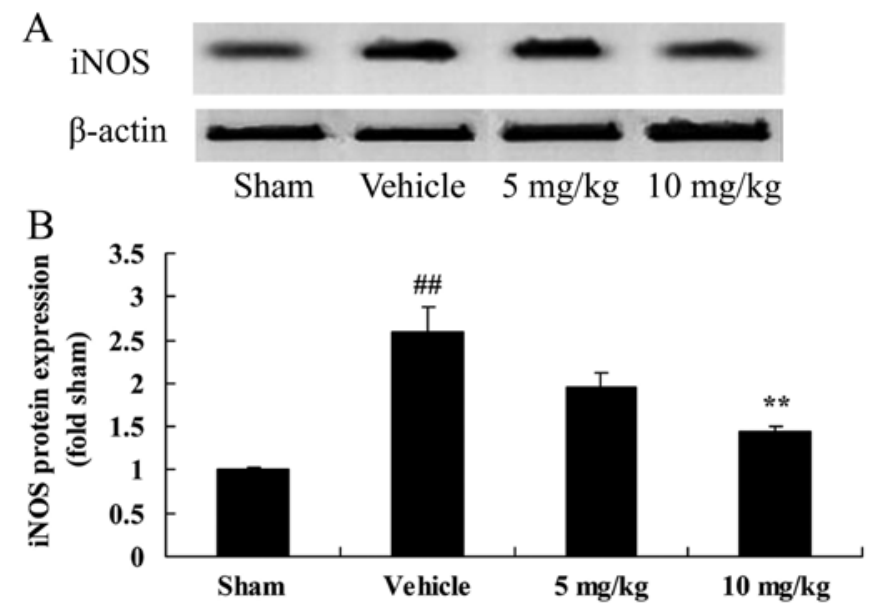

Figure 5. SIRT1 decreases iNOS protein expression in rats with cerebral ischemia reperfusion-induced injury (CIRI). SIRT1 decreased iNOS protein expression, as shown by (A) western blot analysis and (B) statistical analysis of iNOS protein expression in rats with CIRI. Sham, sham-operated group; vehicle, CIRI model rat group; $5 \mathrm{mg} / \mathrm{kg}$, group treated with $5 \mathrm{mg} / \mathrm{kg}$ of SIRT1 activator 3; $10 \mathrm{mg} / \mathrm{kg}$, group treated with $10 \mathrm{mg} / \mathrm{kg}$ of SIRT1 activator 3 . ${ }^{\# \#} \mathrm{p}<0.01$ compared with sham group; ${ }^{* *} \mathrm{p}<0.01$ compared with vehicle group.

CIRI was significantly attenuated by treatment with $10 \mathrm{mg} / \mathrm{kg}$ of SIRT1 activator 3 in the rats with CIRI (Fig. 4).

SIRT1 decreases iNOS protein expression in a rat model of CIRI. The neuroprotective effects of SIRT1 against CIRI may be modulated by various mechanisms, such as the iNOS pathway. Compared with the sham-operated group, CIRI significantly increased iNOS protein expression in the rats with CIRI (Fig. 5). By contrast, treatment with $10 \mathrm{mg} / \mathrm{kg}$ of SIRT1 activator 3 significantly decreased iNOS protein expression in the rats with CIRI (Fig. 5).

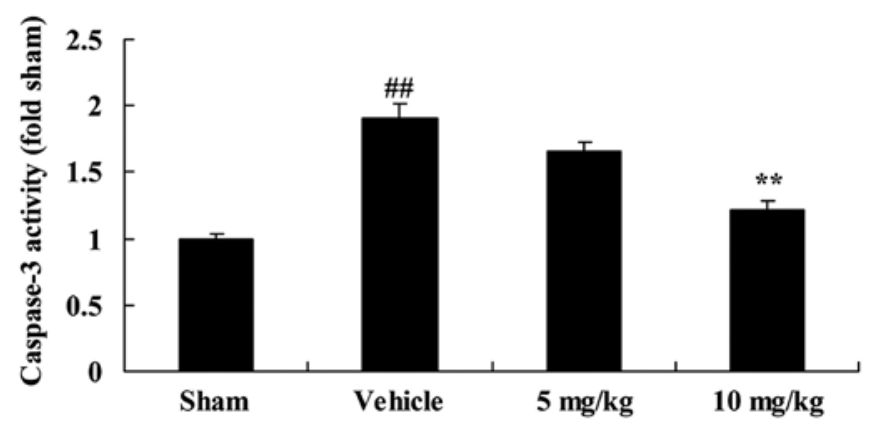

Figure 6. SIRT1 decrases caspase-3 activity in rats with cerebral ischemia reperfusion-induced injury (CIRI). SIRT1 decreased caspase-3 activity as shown by ELISA in rats with CIRI. Sham, sham-operated group; vehicle, CIRI model rat group; $5 \mathrm{mg} / \mathrm{kg}$, group treated with $5 \mathrm{mg} / \mathrm{kg}$ of SIRT1 activator $3 ; 10 \mathrm{mg} / \mathrm{kg}$, group treated with $10 \mathrm{mg} / \mathrm{kg}$ of SIRT1 activator 3. ${ }^{\# \#} \mathrm{p}<0.01$ compared with sham group; ${ }^{* *} \mathrm{p}<0.01$ compared with vehicle group.

SIRT1 decreases caspase- 3 activity in a rat model of CIRI. To examine the role of caspase-3 activity in the effects of SIRT1 on CIRI, caspase-3 activity was measured using an ELISA kit. Indeed, there was a significant increase in caspase-3 activity in the rats with CIRI, compared with those in the sham-operated group (Fig. 6). By contrast, treatment with $10 \mathrm{mg} / \mathrm{kg}$ of SIRT1 activator 3 significantly decreased caspase-3 activity in the rts with CIRI (Fig. 6).

SIRT1 decreases p53 protein expression in a rat model of $C I R I$. To examine the expression profile of p53 protein in the brain tissue samples of rats with CIRI, we performed western blot analysis. The results revealed that the protein expression of p53 was upregulated following CIRI, although this did not reach statistical significance (Fig. 7). Furthermore, treatment with $10 \mathrm{mg} / \mathrm{kg}$ of SIRT1 activator 3 significantly decreased p53 expression in the rats with CIRI (Fig. 7).

SIRT1 increases microRNA-22 expression in a rat model of $C I R I$. We examined the correlation between the effects of SIRT1 and microRNA-22 expression in our rat model of CIRI. The expression of microRNA-22 in the rats with CIRI was significantly inhibited, compared with that in the sham-operated group (Fig. 8). Moreover, the decrease in microRNA-22 expression in the rats with CIRI was reversed by treatment with $10 \mathrm{mg} / \mathrm{kg}$ of SIRT1 activator 3 (Fig. 8).

Downregulation of SIRT1 increases the infarct volume and neurological deficit score in a rat model of CIRI. We then investigated the effects of the downregulation of SIRT1 on infarct volume and neurological deficit score in our rat model of CIRI. As shown by our results, the downregulation of SIRT1 (by treatment with $10 \mathrm{mg} / \mathrm{kg}$ of sirtinol) significantly increased the infarct volume and neurological deficit score in the rats with CIRI, compared with the CIRI model group (Fig. 9).

Downregulation of SIRT1 enhances inflammation in a rat mdoel of CIRI. Subsequently, to further examine the effects of SIRT1 in CIRI brain tissue, we examined the expression of inflammation-related markers in rats with CIRI. The downregulation of SIRT1 (10 mg/kg of sirtinol) significantly 
A
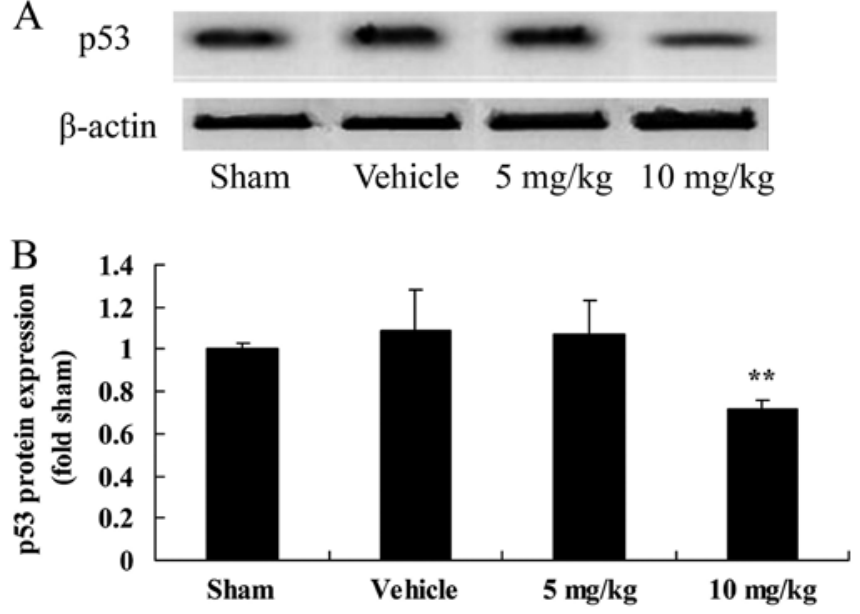

Figure 7. SIRT1 decreases 533 protein expression in rats with cerebral ischemia reperfusion-induced injury (CIRI). SIRT1 decreased $\mathrm{p} 53$ protein expression, as shown by (A) western blot analysis and (B) statistical analysis of p53 protein expression in rats with CIRI. Sham, sham-operated group; vehicle, CIRI model rat group; $5 \mathrm{mg} / \mathrm{kg}$, group treated with $5 \mathrm{mg} / \mathrm{kg}$ of SIRT1 activator $3 ; 10 \mathrm{mg} /$ $\mathrm{kg}$, group treated with $10 \mathrm{mg} / \mathrm{kg}$ of SIRT1 activator $3 .{ }^{\# \#} \mathrm{p}<0.01$ compared with sham group; ${ }^{* *} \mathrm{p}<0.01$ compared with vehicle group.

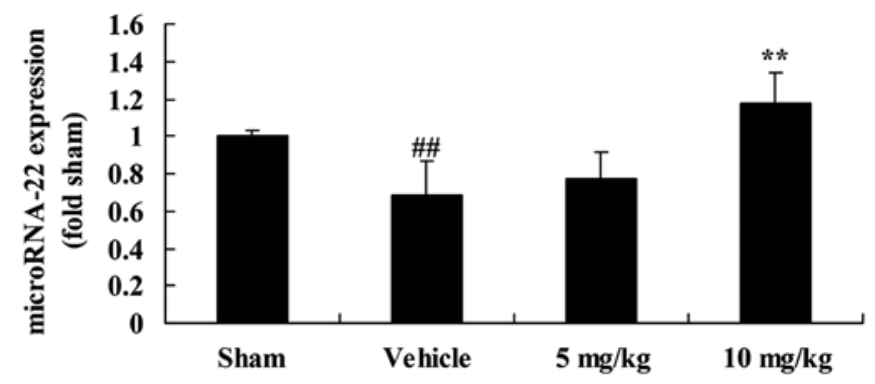

Figure 8. SIRT1 increases microRNA-22 expression in rats with cerebral ischemia reperfusion-induced injury (CIRI). Sham, sham group; vehicle, CIRI model rat group; $5 \mathrm{mg} / \mathrm{kg}$, group treated with $5 \mathrm{mg} / \mathrm{kg}$ of SIRT1 activator 3; $10 \mathrm{mg} / \mathrm{kg}$, group treated with $10 \mathrm{mg} / \mathrm{kg}$ of SIRT1 activator $3 .{ }^{\# \#} \mathrm{p}<0.01 \mathrm{com}-$ pared with sham group; ${ }^{* *} \mathrm{p}<0.01$ compared with vehicle group.

increased the activity of TNF- $\alpha$ and II-6, and inhibited IL-10 activity in the rats with CIRI, compared with the CIRI model group (Fig. 10).

Downregulation of SIRT1 increases COX-2 protein expression in a rat model of CIRI. We then examined COX-2 protein expression following the downregulation of SIRT1 by western blot analysis. The downregulation of SIRT1 (by treatment with $10 \mathrm{mg} / \mathrm{kg}$ of sirtinol) significantly increased the protein expression of COX-2 in the rats with CIRI, compared with the CIRI model group (Fig. 11).

Downregulation of SIRT1 increases iNOS protein expression in a rat model of CIRI. We then further examined the effects of the downregulation of SIRT1 on iNOS protein expression in the rats with CIRI. As shown in Fig. 12, the downregulation of SIRT1 (by treatment with $10 \mathrm{mg} / \mathrm{kg}$ of sirtinol) significantly enhanced the protein expression of iNOS in the rats with CIRI, compared with the CIRI model group.
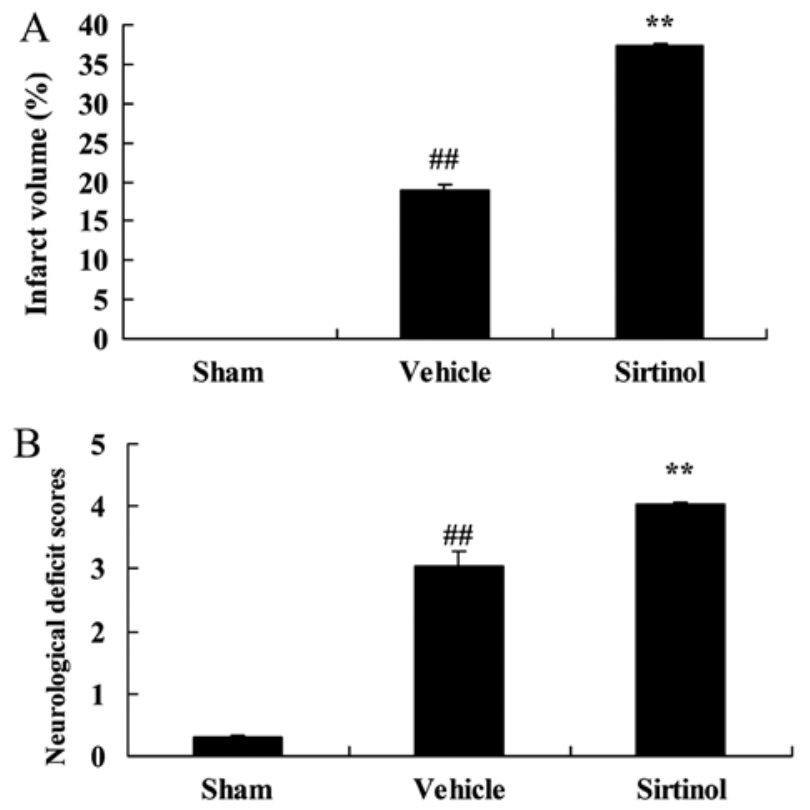

Figure 9. Downregulation of SIRT1 by sirtinol increases the infarct volume and neurological deficit score in rats with cerebral ischemia reperfusioninduced injury (CIRI). Downregulation of SIRT1 increased (A) the infarct volume and (B) the neurological deficit score in rats with CIRI. Sham, sham group; vehicle, CIRI model rat group. ${ }^{\# \#} \mathrm{p}<0.01$ compared with sham group; ${ }^{* *} \mathrm{p}<0.01$ compared with vehicle group.

Downregulation of SIRT1 increases caspase-3 activity in a rat model of CIRI. We then sought to determine whether SIRT1 regulates caspase-3 activity using a SIRT1 inhibitor. According to the results of ELISA, the downregulation of SIRT1 (by treatment with $10 \mathrm{mg} / \mathrm{kg}$ of sirtinol) significantly increased caspase- 3 activity in the rats with CIRI, compared with the CIRI model group (Fig. 13).

Downregulation of SIRT1 increases p53 protein expression in a rat model of CIRI. As p53 signaling is activated with the upregulation of SIRT1, we also examined p53 protein expression in the rats with CIRI following treatment with $10 \mathrm{mg} / \mathrm{kg}$ of sirtinol. As shown in Fig. 14, treatment with $10 \mathrm{mg} / \mathrm{kg}$ of sirtinol significantly increased $\mathrm{p} 53$ protein expression in the rats with CIRI, compared with the CIRI model group.

Downregulation of SIRT1 decreased microRNA-22 expression in a rat model of CIRI. We also performed real-time PCR to examine the effects of the downregulation of SIRT1 on microRNA-22 expression in our rat model of CIRI. Indeed, treatment with $10 \mathrm{mg} / \mathrm{kg}$ of sirtinol significantly inhibited microRNA-22 expression in the rats with CIRI, compared with the CIRI model group (Fig. 15).

\section{Discussion}

During the process of embryonic development, SIRT1 is highly expressed in the brain, spinal cord and dorsal root ganglion (11). In adult brains, SIRT1 is highly expressed in the cortex, hippocampus and epencephalon, while it has a lower expression in aged neurons (14). At present, there is evidence to indicate that SIRT1 plays versatile roles in the central nervous system, such 

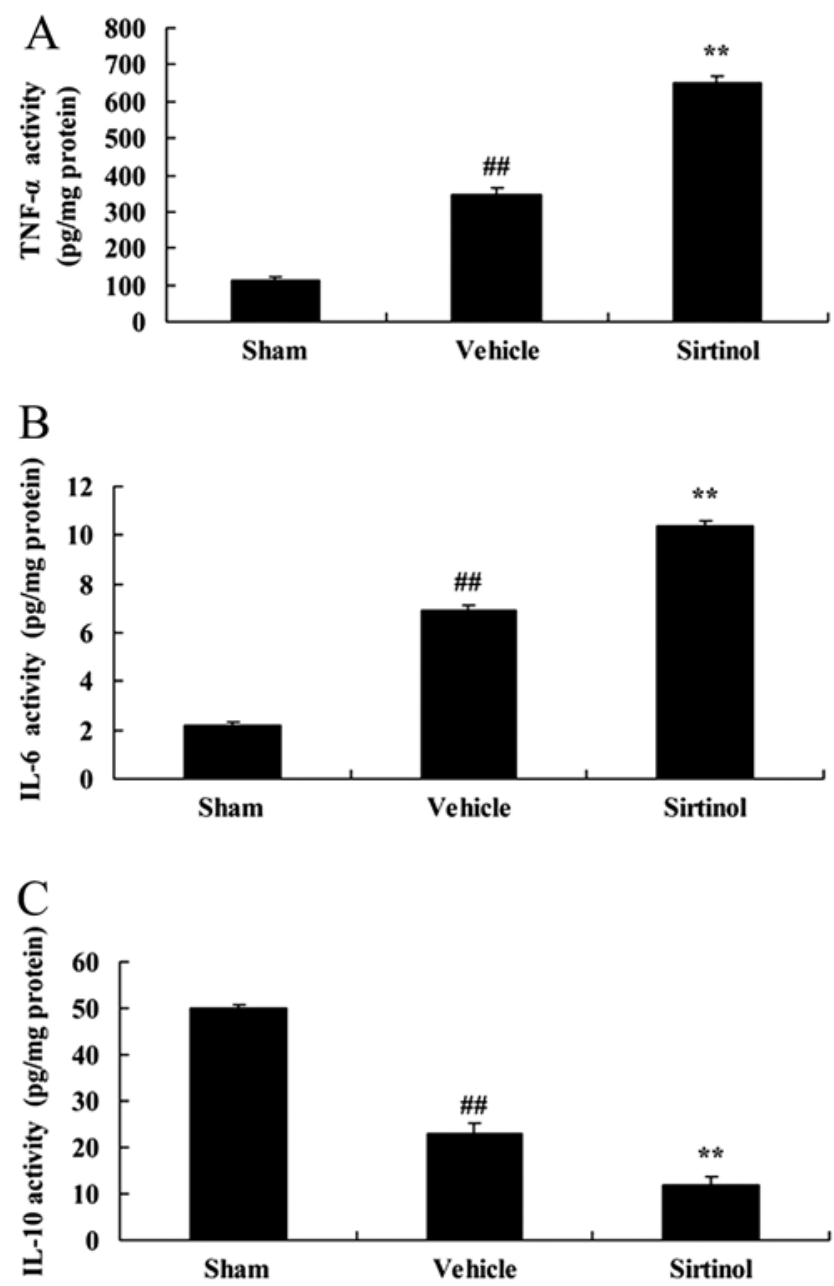

Figure 10. Downregulation of SIRT1 by sirtinol increases inflammation in rats with cerebral ischemia reperfusion-induced injury (CIRI). Downregulation of SIRT1 increased the activity of (A) tumor necrosis factor- $\alpha$ (TNF- $\alpha$ ), (B) interleukin (II)-6 and (C) IL-10 in rats with CIRI. Sham, sham group; vehicle, CIRI model rat group; ${ }^{\# \#} \mathrm{p}<0.01$ compared with sham group; ${ }^{* *} \mathrm{p}<0.01$ compared with vehicle group.

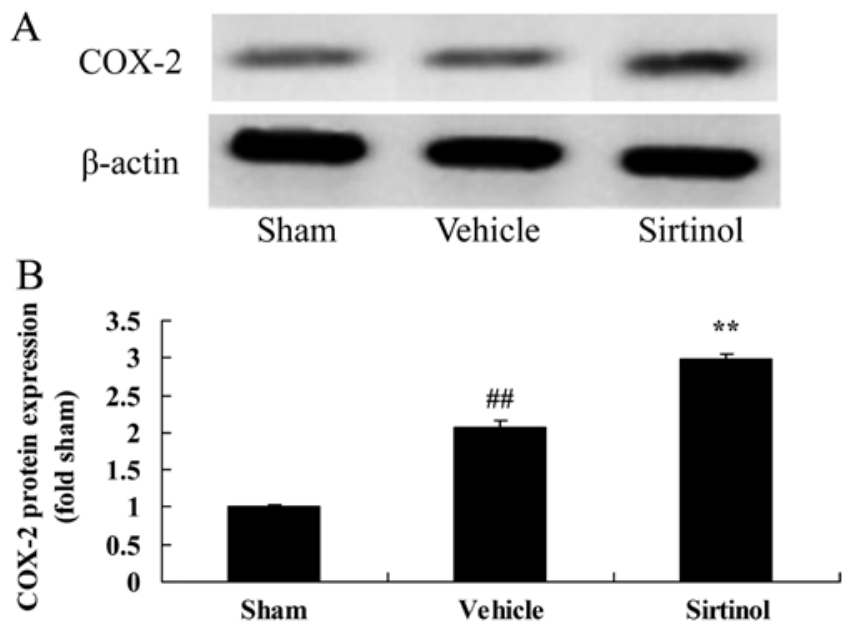

Figure 11. Downregulation of SIRT1 by sirtinol increases COX-2 protein expression in rats with cerebral ischemia reperfusion-induced injury (CIRI). Downregulation of SIRT1 increased COX-2 protein expression, as shown by (A) western blot analysis and (B) statistical analysis of COX-2 protein expression in rats with CIRI. Sham, sham group; vehicle, CIRI model rat group. ${ }^{\# \#} \mathrm{p}<0.01$ compared with sham group; ${ }^{* *} \mathrm{p}<0.01$ compared with vehicle group.
A
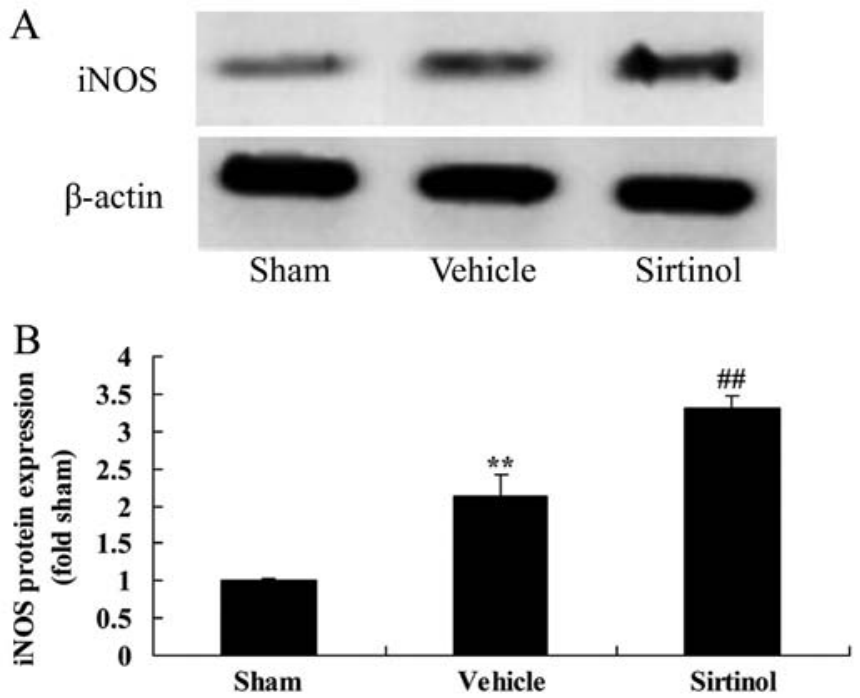

Figure 12. Downregulation of SIRT1 by sirtinol increases iNOS protein expression in rats with cerebral ischemia reperfusion-induced injury (CIRI). Downregulation of SIRT1 increased iNOS protein expression, as shown by (A) western blot analysis and (B) statistical analysis of iNOS protein expression in rats with CIRI. Sham, sham group; vehicle, CIRI model rat group. ${ }^{\# \#} \mathrm{p}<0.01$ compared with sham group; ${ }^{* *} \mathrm{p}<0.01$ compared with vehicle group.

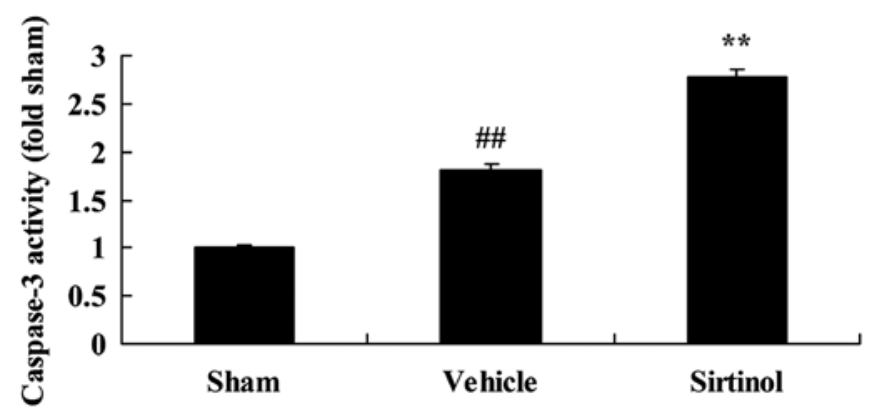

Figure 13. Downregulation of SIRT1 by sirtinol increases caspase-3 activity in rats with cerebral ischemia reperfusion-induced injury (CIRI). Downregulation of SIRT1 increased caspase-3 activity in rats with CIRI. Sham, sham group; vehicle, CIRI model rat group. ${ }^{\#} \mathrm{p}<0.01$ compared with sham group; ${ }^{* *} \mathrm{p}<0.01$ compared with vehicle group.

as neurogenesis and neuroprotection. The increase in the gene expression of SIRT1 can reduce $\beta$-amyloidotic enzyme deposition in rats models of ischemic stroke-induced brain injury and facilitate neuronal survival (13). In addition, SIRT1 plays an important role in the prevention of axonal degenerative disease. SIRT1 can decide the fate of neuronal precursor cells (22). In our study, we found that SIRT1 exerted neuroprotective effects by decreasing the infarct volume and neurological deficit score in a rat model of CIRI. Collectively, our results suggested that SIRT1 may prove to be an important therapeutic target for the treatment of CIRI. Ding et al also suggested that SIRT1 protected against myocardial ischemia-reperfusion injury in diabetic rats (23).

p53 genes are the key regulating point of cellular signaling pathways under a number of pathological conditions and complete adaptive responses by interacting with target genes (24). p53 and its downstream genes play an essential role in cerebral ischemic cell apoptosis (25). Downstream genes of 


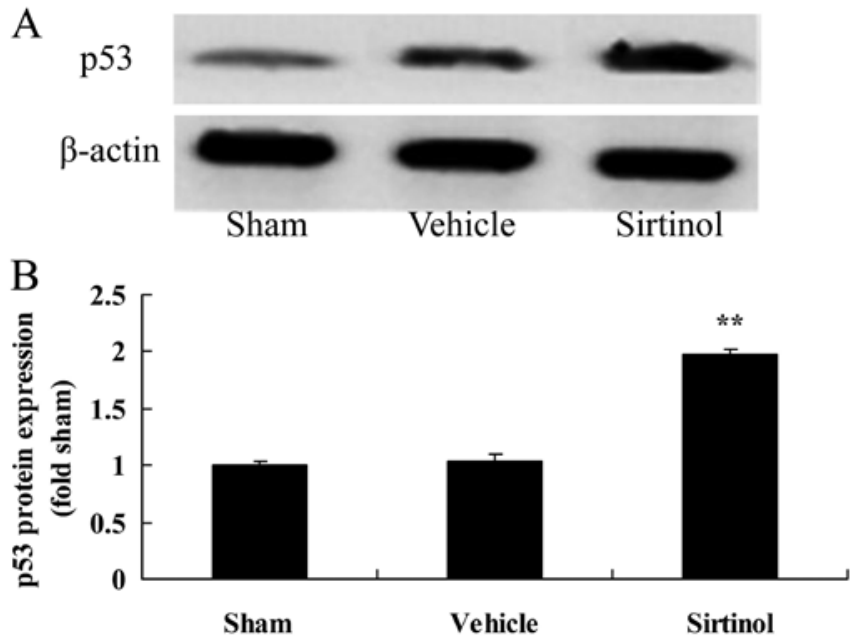

Figure 14. Downregulation of SIRT1 by sirtinol increases p53 protein expression in rats with cerebral ischemia reperfusion-induced injury (CIRI) Downregulation of SIRT1 increased p53 protein expression, as shown by (A) western blot analysis and (B) statistical analysis of $\mathrm{p} 53$ protein expression in rats with CIRI. Sham, sham group; vehicle, CIRI model rat group. ${ }^{\# \#} \mathrm{p}<0.01$ compared with sham group; ${ }^{* *} \mathrm{p}<0.01$ compared with vehicle group.

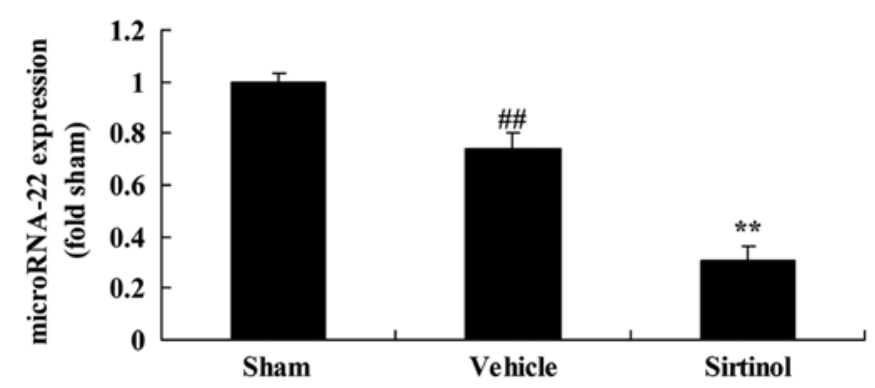

Figure 15. Downregulation of SIRT1 by sirtinol decreases microRNA-22 expression in rats with cerebral ischemia reperfusion-induced injury (CIRI). Sham, sham group; vehicle, CIRI model rat group. ${ }^{\# \#} \mathrm{p}<0.01$ compared with sham group; ${ }^{* *} \mathrm{p}<0.01$ compared with vehicle group.

p53 have two functions, the regulation of the cell cycle and the regulation of cell apoptosis, such as the Bcl-2 family (16). In this study, SIRT1 exerted neuroprotective effects by attenuating p53 protein expression in rats with CIRI. These data suggest that the decreased expression of SIRT1 may contribute to the increased expression of p53 protein in rats with CIRI. Sin et al suggested that the effects of resveratrol on muscle injury were mediated through SIRT1, p53 and caspase-3 in skeletal muscle (26).

With neurotransmitter functions, NO is an important signaling and effector molecule, which extensively participates in physiological and pathological events (27). In the process of CIRI, according to different periods of ischemia and reperfusion, cell types and procedures of NO production, NO can exert dual functions of injury or protection (28). It has been suggested that during CIRI, NOS is the key determinant of whether NO has dual functions (29). At an advanced stage of CIRI, NO generated by iNOS can intensify the toxicity of glutamic acid and result in delayed neuronal damage (27). Particularly, the close integration of iNOS and calmodulin further aggravates DND (30,31). In this study, we found that SIRT1 exerted neuro- protective effects by suppressing iNOS protein expression in rats with CIRI.

COX-2 is a key enzyme in the metabolic pathway of arachidonic acid (AA) (32). Also known as prostaglandin $\mathrm{G} / \mathrm{H}$ synthetase, COX is the catalyzing enzyme of the metabolic transformation of prostaglandins (PGs) and hromboxane (TX) from AA (24). COX exists in tissues in the forms of two subtypes. COX-1 is structural and has certain activities in normal conditions with the basic functions of maintaining physiological processes and the stability of the inner environment (33). COX-2 is inducible and is rarely expressed in normal tissues, while it is highly expressed in tissues during inflammation (34). Many inflammation simulating factors, such as LPS, IL-1, TNF, serum, epidermal growth factor- $\alpha$ and platelet-activating factor can induce the genetic expression of COX-2 and trigger an increase in the levels of PGE2, PGI2 and PGE1 at inflammatory sites (33). COX and its metabolites can aggravate CIRI at the vasculature, blood-brain barrier and neurons (33). The present study clearly demonstrated that SIRT1 exerted neuroprotective effects by suppressing COX-2 protein expression in rats with CIRI. Gano et al reported that the SIRT1 activator, SRT1720, reversed vascular endothelial dysfunction through COX-2 and inflammation in aged mice (35).

Neuro-inflammation during CIRI is key factor giving rise to breakdown, encephaledema and nerve injury. Various measures to relieve the inflammatory response can significantly protect the blood-brain barrier and prevent encephaledema and nerve injury. It has been found that as the dominant ingredient of astragalus, formononetin can markedly reduce the expression of iNOS, IL- $1 \beta$ and TNF- $\alpha$, and decrease the activity of matrix metalloproteinases (MMPs) and the permeability of the bloodbrain barrier, as well as improve nerve function deficit and exert marked neuroprotective effects (36). Our results demonstrated that SIRT1 exerted neuroprotective effect by suppressing the expression of inflammation-related markers in rats with CIRI.

A fair amount of evidence has indicated that apoptosis is strongly linked with nerve cell damage caused by cerebral hypoxia-ischemia (37). Following CIRI, DND may occur at the ischemia-sensitive area and is closely related with molecular mechanisms in the apoptotic process (38). Following focal brain ischemia reperfusion, apoptotic pathways are activated at the ischemia penumbra, resulting in neuronal damage (39). There are three major pathways of cell apoptosis, namely the extrinsic, intrinsic and endoplasmic reticulum (40). As a type of proteolytic enzymes playing key roles, the family of caspases runs through the beginning to the end of the three pathways. As an important family member in the caspase family, caspase-3 plays an essential role in launching, and executing cell apoptosis and cell death $(40,41)$. Our present in vivo results demonstrated that SIRT1 exerted neuroprotective effect by inhibiting caspase- 3 activity in rats with CIRI. Sin et al suggested that the effects of resveratrol on muscle injury were mediated through SIRT1, p53 and caspase-3 in skeletal muscle (26).

MicroRNAs are widely involved in the regulation of nervous system growth and development, as well as in the maintenance of various biological functions (42). MicroRNAs have been found to be expressed in cerebral tissues, and to play a role in the growth and differentiation of cerebral tissues (42). As a complex organ with various cell types, cerebral tissues have different cellular components and synaptic connections at 
different sites (43). Neurons differentiate from progenitor cells. Studies have confirmed that microRNAs promote the differentiation of progenitor cells into certain nerve cells by regulating the expression of target genes and promote differentiated nerve cells maintain innate features (20). In cerebral tissues, cells of different types have individual expression profiles (44). In the present study, we also demonstrated that SIRT1 exerted neuroprotective effects by increasing microRNA-22 expression in rats with CIRI. Zhang et al demonstrated that microRNA-22 functions as a tumor suppressor in renal cell carcinoma through SIRT1 (45).

In conclusion, in the present study, we demonstrated that SIRT1 exerted neuroprotective effects by decreasing the infarct volume and neurological deficit score, suppressing inflammation, decreasing COX-2 and iNOS expression, and inhibiting caspase-3 activity in a rat model of CIRI through the p53/microRNA-22 pathway. Our results open a new line of investigation aimed to determine the neuroprotective effects of SIRT1, which may prove to be an important therapeutic target in CIRI.

\section{References}

1. Bots ML, Ford I, Lloyd SM, Laurent S, Touboul PJ and Hennerici MG; Prevention of Cerebrovascular and Cardiovascular Events of Ischemic Origin With Terutroban in Patients With a History of Ischemic Stroke or Transient Ischemic Attack Vascular Ultrasound Study Investigators: Thromboxane prostaglandin receptor antagonist and carotid atherosclerosis progression in patients with cerebrovascular disease of ischemic origin: A randomized controlled trial. Stroke 45: 2348-2353, 2014.

2. Ibaraki M, Ohmura T, Matsubara K and Kinoshita T: Reliability of CT perfusion-derived CBF in relation to hemodynamic compromise in patients with cerebrovascular steno-occlusive disease: A comparative study with $15 \mathrm{O}$ PET. J Cereb Blood Flow Metab 35: 1280-1288, 2015.

3. Rozanski M, Richter TB, Grittner U, Endres M, Fiebach JB and Jungehulsing GJ: Elevated levels of hemoglobin A1c are associated with cerebral white matter disease in patients with stroke. Stroke 45: 1007-1011, 2014.

4. Liu J, Li J, Yang Y, Wang X, Zhang Z and Zhang L: Neuronal apoptosis in cerebral ischemia/reperfusion area following electrical stimulation of fastigial nucleus. Neural Regen Res 9: 727-734, 2014.

5. Dornbos D III and Ding Y: Mechanisms of neuronal damage and neuroprotection underlying ischemia/reperfusion injury after physical exercise. Curr Drug Targets 13: 247-262, 2012.

6. Szabó C: Physiological and pathophysiological roles of nitric oxide in the central nervous system. Brain Res Bull 41: 131-141, 1996.

7. Ran M, Li Z, Yang L, Tong L, Zhang L and Dong H: Calorie restriction attenuates cerebral ischemic injury via increasing SIRT1 synthesis in the rat. Brain Res 1610: 61-68, 2015.

8. Wang T, Gu J, Wu PF, Wang F, Xiong Z, Yang YJ, Wu WN, Dong LD and Chen JG: Protection by tetrahydroxystilbene glucoside against cerebral ischemia: Involvement of JNK, SIRT1, and NF-kappaB pathways and inhibition of intracellular ROS/RNS generation. Free Radic Biol Med 47: 229-240, 2009.

9. Zhang Q, Wang SY, Fleuriel C, Dominique L, Rocheleau JV, Piston DW and Goodman RHL: Metabolic regulation of SIRT1 transcription via a HIC1:CtBP corepressor complex. Proc Natl Acad Sci USA 112: E819, 2015.

10. Martin A, Tegla CA, Cudrici CD, Kruszewski AM, Azimzadeh P, Boodhoo D, Mekala AP, Rus V and Rus H: Role of SIRT1 in autoimmune demyelination and neurodegeneration. Immunol Res 61: 187-197, 2015.

11. Wang L, Zhang L, Chen ZB, Wu JY, Zhang X and Xu Y: Icariin enhances neuronal survival after oxygen and glucose deprivation by increasing SIRT1. Eur J Pharmacol 609: 40-44, 2009.

12. Santo EE and Paik J: FOXO3a \& haematopoietic stem cells: Goodbye PI3K, hello SIRT1? Cell Cycle 15: 879-880, 2016.
13. Miao Y, Zhao S, Gao Y, Wang R, Wu Q, Wu H and Luo T: Curcumin pretreatment attenuates inflammation and mitochondrial dysfunction in experimental stroke: The possible role of Sirt1 signaling. Brain Res Bull 121: 9-15, 2016.

14. Meng Z, Li J, Zhao H, Liu H, Zhang G, Wang L, Hu HE, Li DI, Liu M, Bi F, et al: Resveratrol relieves ischemia-induced oxidative stress in the hippocampus by activating SIRT1. Exp Ther Med 10: 525-530, 2015.

15. Araki T and Kogure K: Prevention of delayed neuronal death in gerbil hippocampus by a novel vinca alkaloid derivative (vinconate). Mol Chem Neuropathol 11: 33-43, 1989.

16. Guo YB, Bao XJ, Xu SB, Zhang XD and Liu HY: Honokiol induces cell cycle arrest and apoptosis via p53 activation in H4 human neuroglioma cells. Int J Clin Exp Med 8: 7168-7175, 2015.

17. Leak RK, Zhang L, Luo Y, Li P, Zhao H, Liu X, Ling F, Jia J, Chen J and Ji X: Peroxiredoxin 2 battles poly(ADP-ribose) polymerase 1- and p53-dependent prodeath pathways after ischemic injury. Stroke 44: 1124-1134, 2013.

18. Hu Y, Deng H, Xu S and Zhang J: MicroRNAs regulate mitochondrial function in cerebral ischemia-reperfusion injury. Int J Mol Sci 16: 24895-24917, 2015.

19. Ni J, Wang X, Chen S, Liu H, Wang Y, Xu X, Cheng J, Jia J and Zhen X: MicroRNA let-7c-5p protects against cerebral ischemia injury via mechanisms involving the inhibition of microglia activation. Brain Behav Immun 49: 75-85, 2015.

20. Wang C, Pan Y, Cheng B, Chen J and Bai B: Identification of conserved and novel microRNAs in cerebral ischemia-reperfusion injury of rat using deep sequencing. J Mol Neurosci 54: 671-683, 2014.

21. Yang ZB, Zhang Z, Li TB, Lou Z, Li SY, Yang H, Yang J, Luo XJ and Peng J: Upregulation of brain-enriched miR-107 promotes excitatory neurotoxicity through downregulation of glutamate transporter-1 expression following ischaemic stroke. Clin Sci (Lond) 127: 679-689, 2014.

22. Kalaivani P, Ganesh M, Sathiya S, Ranju V, Gayathiri V and Saravana Babu C: Alteration in bioenergetic regulators, SirT1 and Parpl expression precedes oxidative stress in rats subjected to transient cerebral focal ischemia: molecular and histopathologic evidences. J Stroke Cerebrovasc Dis 23: 2753-2766, 2014.

23. Ding M, Lei J, Han H, Li W, Qu Y, Fu E, Fu F and Wang X: SIRT1 protects against myocardial ischemia-reperfusion injury via activating eNOS in diabetic rats. Cardiovasc Diabetol 14: 143 , 2015.

24. Chai YS, Hu J, Lei F, Wang YG, Yuan ZY, Lu X, Wang XP, Du F, Zhang D, Xing DM, et al: Effect of berberine on cell cycle arrest and cell survival during cerebral ischemia and reperfusion and correlations with p53/cyclin D1 and PI3K/Akt. Eur J Pharmacol 708: 44-55, 2013.

25. Tu YF, Lu PJ, Huang CC, Ho CJ and Chou YP: Moderate dietary restriction reduces p53-mediated neurovascular damage and microglia activation after hypoxic ischemia in neonatal brain. Stroke 43: 491-498, 2012

26. Sin TK, Yung BY, Yip SP, Chan LW, Wong CS, Tam EW and Siu PM: SIRT1-dependent myoprotective effects of resveratrol on muscle injury induced by compression. Front Physiol 6: 293, 2015.

27. Shen J, Ma S, Chan P, Lee W, Fung PC, Cheung RT, Tong Y and Liu KJ: Nitric oxide downregulates caveolin-1 expression in rat brains during focal cerebral ischemia and reperfusion injury. J Neurochem 96: 1078-1089, 2006.

28. Kidd GA, Hong H, Majid A, Kaufman DI and Chen AF: Inhibition of brain GTP cyclohydrolase I and tetrahydrobiopterin attenuates cerebral infarction via reducing inducible NO synthase and peroxynitrite in ischemic stroke. Stroke 36: 2705-2711, 2005.

29. Heeba GH and El-Hanafy AA: Nebivolol regulates eNOS and iNOS expressions and alleviates oxidative stress in cerebral ischemia/reperfusion injury in rats. Life Sci 90: 388-395, 2012.

30. Chen YF, Wang YW, Huang WS, Lee MM, Wood WG, Leung YM and Tsai HY: Trans-cinnamaldehyde, an essential oil in cinnamon powder, ameliorates cerebral ischemia-induced brain injury via inhibition of neuroinflammation through attenuation of iNOS, COX-2 expression and NFk-B signaling pathway. Neuromolecular Med 18: 322-333, 2016.

31. Ren B, Zhang YX, Zhou HX, Sun FW, Zhang ZF, Wei Z, Zhang CY and Si DW: Tanshinone IIA prevents the loss of nigrostriatal dopaminergic neurons by inhibiting NADPH oxidase and iNOS in the MPTP model of Parkinson's disease. J Neurol Sci 348: 142-152, 2015. 
32. Han X, Li H, Su L, Zhu W, Xu W, Li K, Zhao Q, Yang H and Liu H: Effect of celecoxib plus standard chemotherapy on serum levels of vascular endothelial growth factor and cyclooxygenase-2 in patients with gastric cancer. Biomed Rep 2: 183-187, 2014.

33. Vaibhav K, Shrivastava P, Javed H, Khan A, Ahmed ME, Tabassum R, Khan MM, Khuwaja G, Islam F, Siddiqui MS, et al: Piperine suppresses cerebral ischemia-reperfusion-induced inflammation through the repression of COX-2, NOS-2, and $\mathrm{NF}-\kappa \mathrm{B}$ in middle cerebral artery occlusion rat model. Mol Cell Biochem 367: 73-84, 2012.

34. Gaur V and Kumar A: Effect of nonselective and selective COX-2 inhibitors on memory dysfunction, glutathione system, and tumor necrosis factor alpha level against cerebral ischemia reperfusion injury. Drug Chem Toxicol 35: 218-224, 2012.

35. Gano LB, Donato AJ, Pasha HM, Hearon CM Jr, Sindler AL and Seals DR: The SIRT1 activator SRT1720 reverses vascular endothelial dysfunction, excessive superoxide production, and inflammation with aging in mice. Am J Physiol Heart Circ Physiol 307: H1754-H1763, 2014.

36. Sun K, Fan J and Han J: Ameliorating effects of traditional Chinese medicine preparation, Chinese materia medica and active compounds on ischemia/reperfusion-induced cerebral microcirculatory disturbances and neuron damage. Acta Pharm Sin B 5: 8-24, 2015.

37. Yu H, Liu P, Tang H, Jing J, Lv X, Chen L, Jiang L, Xu J and Li J: Oleuropein, a natural extract from plants, offers neuroprotection in focal cerebral ischemia/reperfusion injury in mice. Eur J Pharmacol 775: 113-119, 2016.

38. Wang J, Wang P, Li S, Wang S, Li Y, Liang N and Wang M: Mdivi-1 prevents apoptosis induced by ischemia-reperfusion injury in primary hippocampal cells via inhibition of reactive oxygen species-activated mitochondrial pathway. J Stroke Cerebrovasc Dis 23: 1491-1499, 2014.
39. Zhang JF, Shi LL, Zhang L, et al: MicroRNA-25 negatively regulates cerebral ischemia/reperfusion injury-induced cell apoptosis through Fas/FasL pathway. J Mol Neurosci 58: 507-516, 2016.

40. Yaidikar L and Thakur S: Punicalagin attenuated cerebral ischemia-reperfusion insult via inhibition of proinflammatory cytokines, up-regulation of Bcl-2, down-regulation of Bax, and caspase-3. Mol Cell Biochem 402: 141-148, 2015.

41. Tao T, Li CL, Yang WC, Zeng XZ, Song CY, Yue ZY, Dong H and Qian H: Protective effects of propofol against whole cerebral ischemia/reperfusion injury in rats through the inhibition of the apoptosis-inducing factor pathway. Brain Res 1644: 9-14, 2016.

42. Stary CM, Xu L, Sun X, Ouyang YB, White RE, Leong J, Li J, Xiong $X$ and Giffard RG: MicroRNA-200c contributes to injury from transient focal cerebral ischemia by targeting Reelin. Stroke 46: 551-556, 2015.

43. Liu P, Zhao H, Wang R, Wang P, Tao Z, Gao L, Yan F, Liu X, Yu S, Ji X, et al: MicroRNA-424 protects against focal cerebral ischemia and reperfusion injury in mice by suppressing oxidative stress. Stroke 46: 513-519, 2015.

44. Brandenburger T, Grievink H, Heinen N, Barthel F, Huhn R, Stachuletz F, Kohns M, Pannen B and Bauer I: Effects of remote ischemic preconditioning and myocardial ischemia on microRNA-1 expression in the rat heart in vivo. Shock 42: 234-238, 2014.

45. Zhang S, Zhang D, Yi C, Wang Y, Wang H and Wang J: MicroRNA-22 functions as a tumor suppressor by targeting SIRT1 in renal cell carcinoma. Oncol Rep 35: 559-567, 2016. 